\title{
The device to improve the working element of the rotary trench excavator in the production process of breaking a main pipeline
}

\author{
Asvar Akhmedov, ${ }^{1, *}$ \\ ${ }^{1}$ Volgograd State Technical University, 400005, Lenin avenue, 28, Volgograd, Russia
}

\begin{abstract}
The article develops a device for the rotary trench excavator. The device will enable to break an underground major pipeline from one of the side faces and above the top surface of the pipe in one operation. The design of the device enables to use it in any climatic conditions. The application of the improved device attached to the rotary trench excavator enables to enforce the structure of the construction and repair spread.
\end{abstract}

Today, the construction and the major repairs of the linear section of major pipeline require some "breakthrough" inventions to enhance technical and economic performance. The oil and gas industry has a problem that lies in the fact that the lion's share of the presently exploited pipelines needs immediate overhaul operation. To this end, it is urgent to develop new innovations devices to improve technological processes. Due to the improvement of technological processes and operations it is possible to reach better technical and economic performance. This will enable to improve the situation with the state of main pipelines.

The application of continuous-type machines cuts the duration of stripping works by 10 to 15 times which undoubtedly is profitable for companies doing the overhaul operations.

Today, there is no fleet of rotary trench excavators at the organizations performing the overhaul works for the main pipelines [1-3]. However, our country goes on to produce the rotary trench excavators. The rotary trench excavators are made and sold in this country up to now.

Search for power efficient technologies on the major repairs of main pipelines is being done in many countries and this is connected not only with the fact of increasing volumes of worn-out pipes, but also with the situation when pipeline mains go through various climatic and natural conditions [4-8].

A machine is known for the layer-by-layer stripping of the main pipeline [9] that has milling tools as a working element. To break the pipe using this machine, it is necessary to do several passes which increases the unproductive movements.

The disadvantage of the invention is the fact that after the digging machine operation, it is difficult to do further repair works and there is no access for machines and mechanisms since the soil lifted with the buckets of working elements is falling on the conveyors and brought to the burrows at the both sides of the trench which requires the equipment to trim the excavated soil along the whole length of the stripped section of the pipeline.

A rotary trench excavator is known with a special working element [10] consisting of three rotors with buckets mounted on the frame suitable for movement relative to one another vertically. The present ability to move rotors vertically relative to one another requires a powerful framework and a strong hydraulics which increases the cost of the working element.

A single-bucket excavator is known [11] with a conveyor and a blade on it which enables to remove the soil outside the machine as stripping moves forward. However, the given excavator cannot strip the trench without tip trucks taking the soil from the conveyor.

There is also another technology [12] consisting of the basic machine with the mounted special working element enabling to strip the pipeline from both lateral sides and along the lower moving line. According to the technology, to strip a pipeline, it is necessary to strip the whole pipeline section under repair using any digging machine up to the upper line which makes costs of the earthworks higher. Apart from this, the design of the working element prevents from moving the soil to only one side when stripping the pipeline which involves the need to level one of the trench edges to move a construction train. This results in reducing the technical and economic performance.

From the existing technology level, we know the inventor's certificate [13] lying in the fact that the working element of the machine is two rotors parallel fixed in the vertical plane suitable for changing the distance between them. There is a "dumping knife" between the rotors cutting the soil along the top of the pipe as the stripping moves forward guiding it onto the rotating rotors on both lateral sides; they move the soil to the disposable area via a conveyor.

Despite the fact that the given working element can be applied for stripping pipelines of several diameters due to the rotors' readjustment, the use for all the

\footnotetext{
* Corresponding author: asvar05@mail.ru
} 
diameter types is limited by the framework sizes. To strip pipes of greater diameters, the change of the framework for another with greater sizes is required. The installation and the following adjustment require the application of lifting facilities and hand labor.

There exists one more invention [14], the working element of the excavator for the pipeline break including a rotor with the buckets and the shortened middle section, and the buckets of the extreme sections are made with the slope angle to prevent the shock impacts on the pipeline walls. The given technological solution is directed at breaking the pipeline from the lateral sides and from the pipe top in one passage of the machine. The drawback of the invention is undoubtedly the inability to use it in all the pipeline diameters which reduces its demand and universal application.

In [15] the stripping of the pipeline is carried out by means of the simultaneous movement of the column of technical facilities and mechanisms connected with one another by cables. There is a tow truck at the front of the column to which a bulldozer is attached by a cable cutting the vegetable mold and moving it to both sides of the stripped pipeline with the help of the blade and the ripper arrangement in the direction of its travel. The second tow truck is fixed to the bulldozer by ropes which pulls the second ripper which does its work and moves apart the mineral soil above the pipeline by its "blades-molds" to both sides from the axis of the stripped trench. The pipe-layers moving in step with the tow trucks and the bulldozer provide the pipeline's lifting with the trolley pipe holders, herewith, the carrying crossbeam is joined to the ripper of the bulldozer which ensures the removal of the soil rests from the upper part of the pipe and its lifting from the foundation level by the trolley pipe holder. Plow-type device behind the multi-bank trolley holder removes the soil to the trench and surfaces the ground onto which the lifted pipeline is laid due to the reverse turning of the plowshares in its forward motion parallel to the ripping and moving the soil apart with the first and the second rippers.

Thus, in the known technique, the break is done due to the simultaneous motion of all the technical facilities and creation of considerable pulling force.

Among the great variety of the existing working elements of the rotary trench excavators for stripping a linear section of main pipelines, there are a multitude of technical solutions with considerable drawbacks. Many of them are outdated and require the upgrading and improving with regard to modern achievements in science and technology.

With regard to the studied problems and drawbacks, a special rotary trench excavator was proposed the working element of which has been modernized. Further, we will consider the proposed device in more details taking into account its applicability.

The device can be used while stripping during the major repairs of main pipeline as well as in disassembling the decommissioned pipes.

The proposed innovative device enables to break a pipeline in the broad range of diameters from 520 to 1620 included. The device is a hitch attachment and is connected to the base machine of the rotary trench excavator that is strengthened with an extra space frame which adds an extra rigidity and stability to the working element.

The developed device can significantly facilitate the break of the main pipeline. The application of the hitch attachment along with the working element of the rotary excavator enables to additionally enhance the productivity and efficiency in the work of the digging machine.

The increase in the values of technical and economic indexes can attract specialists to the developed device. The increase in these indexes and the improvement of technical characteristics of the existing digging machines will enable to save considerable financial means and so their introduction into the production will give a short payback period.

The short payback period is the key to the rapid spread in the market and mainstreaming in those branches where it is required to break a pipeline for repair and maintenance works or to change the pipe's wall.

The proposed device along with the working element of the rotary trench excavator enables to bring the technological process to a new qualitative level.

The device is designed for enlarging the capabilities of the rotary trench excavator due to improvement of its working element. With the improved rotary trench excavator, the pipeline section under repairs can be stripped in one passage.

The device is made as a spatial one, suitable for quick installation, removal due to a special removable connection. Besides, the device can be easily repaired and moved due to its competent and compact performance.

The use of the device enables to bring the technological process of breaking the linear section of main pipelines to a new qualitative level.

Based on the analysis of the existing technical solutions [1-16] for improving the technology of breaking the underground main pipeline using the rotary trench excavator, a special innovative device has been developed. The proposed device is designed on the basis of the common mass-produced rotary trench excavator.

The target of the device is to enlarge technological capabilities of the rotary trench excavator with the simultaneous reduction of labor intensity for earthworks due to the increase of the operating efficiency coupled with the machine's working element.

In order to reach the target, the following problems are determined and put:

- to develop a device which will help to break the main pipeline from the lateral side and above the top plane in one passage;

- to develop a technical solution for the device that will move the soil to one side in the direction of the machine movement;

- to develop a technical solution for the device enabling to use the device for different diameters of main pipelines.

The device consists of a space frame 1that is fixed to the base mounting of the basic machine 2 of the rotary excavator. The space frame 1 consists of the threaded 
guide rods состоит 3 one each of which the drive components 4 are mounted with the electric motors 5 . The drive components 4 with the electric motors 5 are located to move along the rods 3 . The milling tools 6 are hardly connected to the drive components 4 . Herewith, the number of the milling tools is selected based on the width of the stripped trench 7 and the diameter of the main pipeline 8 . The milling tools are mounted rigidly to the rods 3 with their upper and lateral sides which prevents them from shifting in the stripping process. The rods 3 with the milling tools are mounted on the metal frame by which they are connected with the base machine 2 from one lateral side, and from the other side they are connected to the rubber tired base mounting. To move the milling tools 8 in the traverse direction, some extra threaded rods 9 are mounted to the rods 3 on which there are extra drive components 10 with the electric motors 11 for the transfer purposes (Fig. 1.- 6).

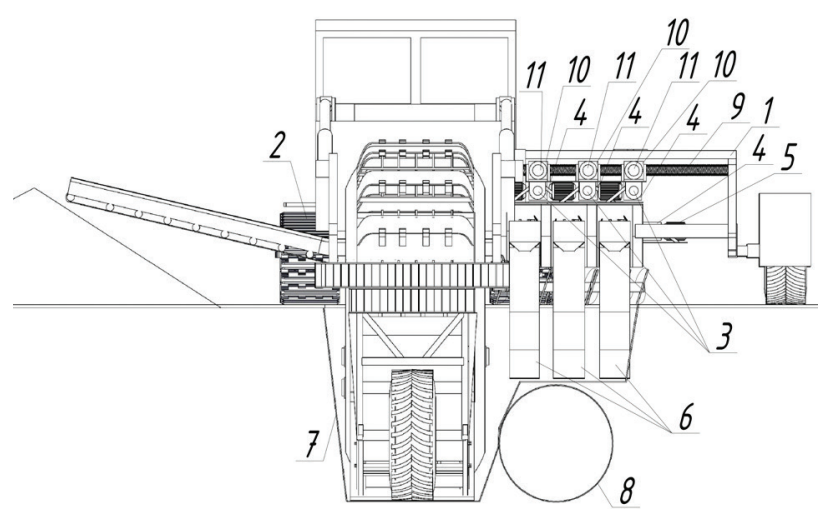

Fig. 1. Caption of the Figure 1. Below the figure: $1-$ space frame , 2 - base machine, 3 - threaded guide rod, 4 - drive, 5 electric motor, 6 - milling tools, 7 - trench, 8 - pipeline, 9 extra rod, 10 - extra drive, 11 -electric motor

The device operates in coordination with the rotary trench excavator in the following way. At first, the rotary trench excavator is located at the start of the stripping segment of the main pipeline. Hereafter, the device is connected to the lateral side of the base machine. The device is designed so that it can be connected both to the one and to the other lateral side of the rotary trench excavator. Thereafter, the milling tools are moved to the needed location and the break (stripping) starts. The milling tools are located offset relative to one another which enables to get the "effect of the ground failure". Then the rotor with the buckets is buried so that it is to one side from the stripped segment of the pipe. When the rotor reaches the design reference mark of the trench bottom, the milling tools and the screw conveyers are switched on. While running the milling tools, the screw conveyers are brought to the rotational motion which moves the soil lifted by the drilling tools to the rotating rotor with the buckets. In their turn, the moving rotor's buckets will take the soil and move it to the stacker conveyer from which it will go to the disposal area. The proposed device enables to carry out the stripping of the main pipeline from one of the lateral sides and from the top side of the pipe in one passage of the rotary trench excavator.
The application of chain milling tools in the device's design enables to break the pipeline above the top side of the pipe in the wide interval in depth. The adjustment of the milling tools location is done from the remote controller in the operator's cabin. And the visual control over the location of the device and the working element is carried out by video cameras fixed on the base machine. If necessary to relocate the rotary trench excavator to a new place of the trench break, the device is removed and transferred on the individual basis.

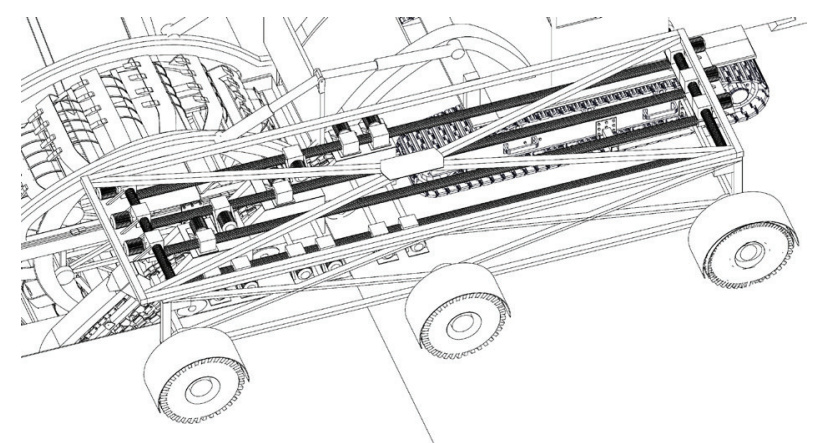

Fig. 2. Space simulation model of the device.

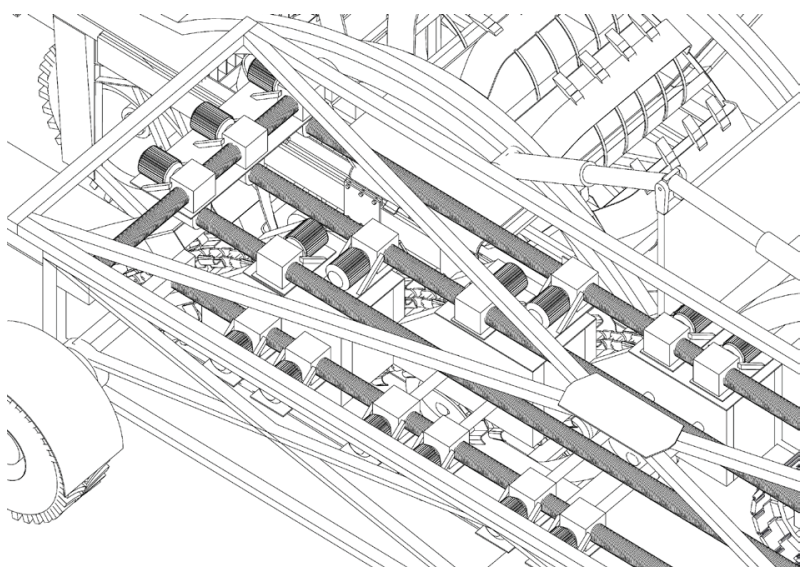

Fig. 3. Dimensional view of the connection for the guide rods with an additional rod.

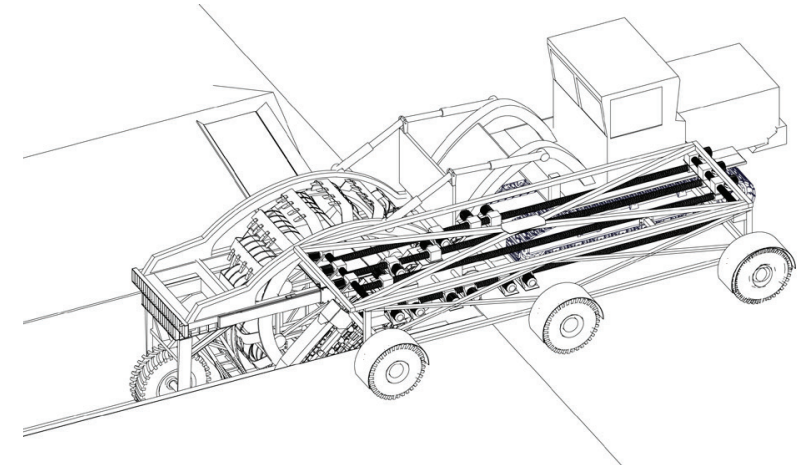

Fig. 4. Lateral dimensional view of the device in the working order while breaking a main pipeline. 


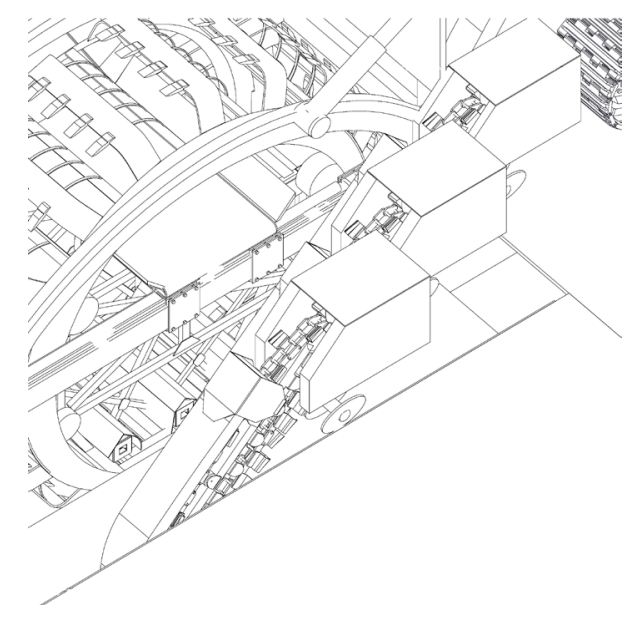

Fig. 5. Milling tools fixed on the devices with the shift relative to one another to get the "effect of the ground failure".

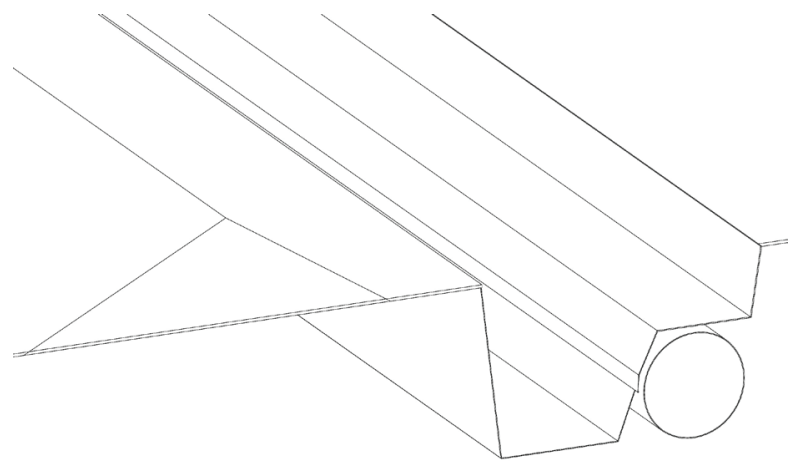

Fig. 6. General space view of the trench after the stripping.

Thus, the device for stripping the main pipeline compared to the known technical solutions enables to reduce the amount of the excavated soil due to the fact that the break is carried out from one lateral side and above the pipeline, coupled with the simultaneous cutting of machines for the pipeline break. Herewith, the time consumption to get the machine with the device into working trim is also cut, that is a new technical result of the proposed technical solution.

To prevent the damage of the main pipeline while breaking it as well as to exclude the chance of milling tools damage, it is proposed to place two fixed stops on the rear wheel assembly and inside the rotor with the buckets.

Both fixed stops 1 and 2 are brought into motion by horizontally mounted jacks. The fixed stops are made of metal based rubber. The fixed stops 1 and 2 are rigidly fixed on the working element of the rotary trench excavator.

The fixed stop 1 located on the rear support is suitable for altitude adjustment, to have a possibility to change its altitude depending upon the diameter of the stripped pipeline. The fixed stops are brought into motion with the horizontally fixed jacks 3 and the guide rods 4 which prevent the shift of fixed stops (Fig. 7-9). Besides, a sensor is mounted on each of the fixed stops that will send a signal to the operator's cabin in case of pressure boost in the hydraulic fluid in the jacks. Since when the fixed stop touches the surface, there appears a pressure loads which can sharply increase the pressure in the hydraulic cylinders. According to the sound signal for the operator he can correct the position of the working element and go on to break the pipeline.

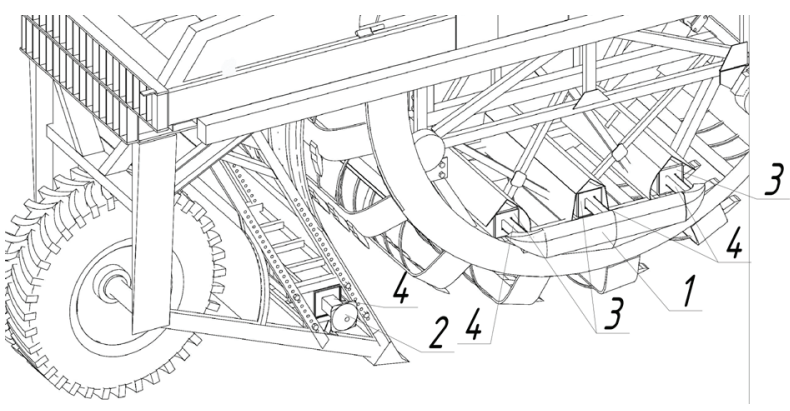

Fig. 7. General space view of the fixed stop system. 1, 2 - fixed stops, 3 - horizontally fixed jacks, 4 - guide rods

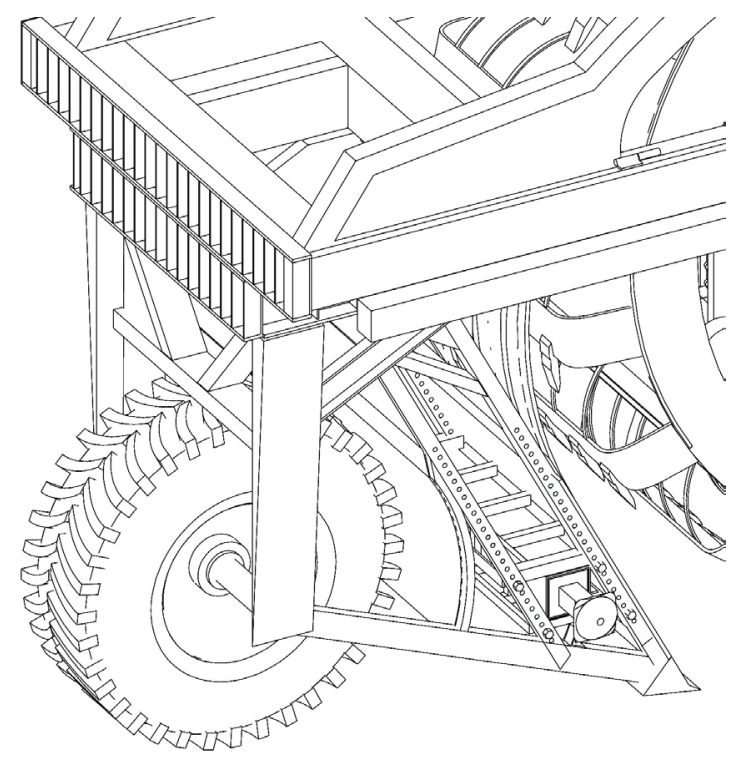

Fig. 8. 3D configuration of a fixed stop mounted on the rear wheel assembly of the rotary trench excavator.

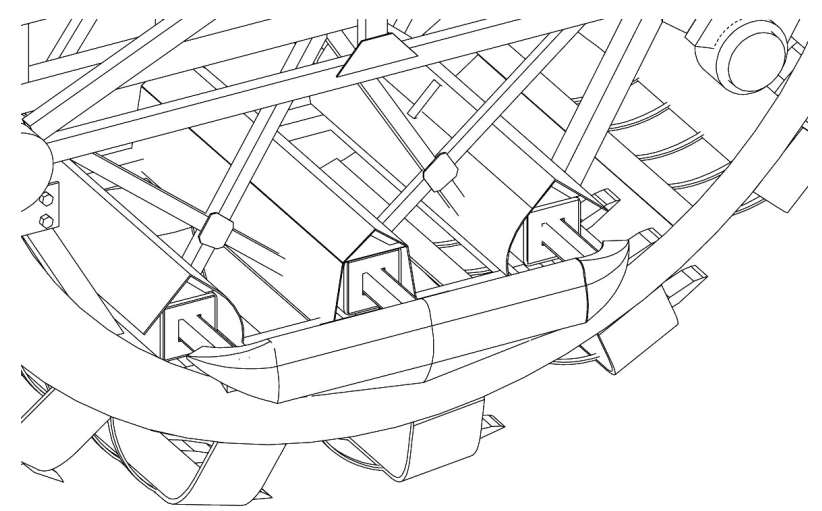

Fig. 9. 3D configuration of a fixed stop placed inside the rotor with buckets.

The proposed system of fixed stops along with the device for stripping the main pipeline help to carry out the break of the main pipeline carefully without damaging it. 


\section{References}

1. A.S. Shatskiy, On the issue of using rotary trench excavators in Russian pipeline construction, Truboprobodniy transport, No. 2(36). pp. 28-31 (2013)

2. A.S. Shatskiy, G.R. Gabelaya, On the issue of optimization of earthworks building pipelines under the conditions of the Extreme North, Troboprovodniy transport, No. 3 (31), pp. 17-21 (2012)

3. S.I.Larin, M.Z. Alshits, The rotary trench excavator ETR309, Mashiny i oborudovaniye, No. 1. P. 21-22 (2009)

4. G. Ferris, S. Newton, M. Porter, Proceedings of the 11th International Pipeline Conference, 2016, 2, UNSP V002T07A001, (2017)

5. F. Mosleh, T. Zayed, MS El-Abbasy, Journal of Performance of Constructed Facilities, 30 (Iss. 6), 04016037 (2016)

6. M. Elchalakani, Journal of Constructional Steel Research, 125, 26 (2016)

7. R. Shahandeh, H. Showkati, Journal of Constructional Steel Research, 121, 237 (2016)

8. V. Ivanov, M. Zykov, V. Rybin, S. Plotnikov, XV International Conference Topical Problems of Architecture, Civil Engineering, Energy Efficiency and Ecology - 2016, 73, UNSP 01024 (2016)

9. D.F. Ihsanov, V.L. Buzhinskij, A. G. Gumerov, A.A. Bikbulatov, V.M. Murov, Z.K. Berdnikova

Patent RU, No. 887740, 2006.

10. Alexandr V. Bykov e.a., Machine for digging into the lower layers of the ground. Patent US, No. 6418646 B1, 2002.

11. Gareth J.T. e.a.,. Patent UK, no. 2435653 (2006)

12. Evgeny P. Kovalev e.a. Patent US, No. 4976051 (1990)

13. Pierluigi Zanin., e.a. Patent US, No. 5601383 (1997)

14. Z.K. Berdnikova, Z.H. Valeev, V.E. Gubin, F.H. Ismagilova, O.N. Kanavin, E.K. Kondrat'eva

Patent SSSR, No. 257355 (1990)

15. A.M. Muhametshin e.a., Patent RU, No. 2389930, (2010)

16. Danny R. MCCARTY e.a., Excavating systems and methods. Patent US, No. 2013/0025169 A1 (2013) 